\title{
EFICIÊNCIA DE INSETICIDAS NATURAIS E TIAMETOXAM NO CONTROLE DE TRIPES EM VIDEIRA E SELETIVIDADE PARA INIMIGOS NATURAIS
}

\author{
LUIZ RONALDO NALI * \\ FLÁVIA RABELO BARBOSA ** \\ CARLOS ALFREDO LOPES DE CARVALHO *** \\ JACKSON BONFIM CARVALHO DOS SANTOS ****
}

\begin{abstract}
O objetivo deste trabalho foi avaliar a eficiência de produtos naturais e do tiametoxam no controle de tripes em videira e seus efeitos sobre inimigos naturais. $O$ experimento foi instalado em plantio comercial, no município de Juazeiro-BA (Brasil), em parreiral da variedade Benitaka, com 5 anos de idade, irrigado por microaspersão. Adotou-se 0 delineamento experimental em blocos ao acaso, com 4 repetições e 5 tratamentos, sendo: 1 . Nim-I-Go a $0,5 \%$; 2. Rotenat a $0,5 \%$; 3 . Bio Alho a $0,3 \% ; 4$. tiametoxam 250WG (20 g de produto comercial/100L) e 5. testemunha (sem inseticida). Foram utilizadas 12 plantas por tratamento, sendo avaliados o número de tripes e de inimigos naturais por inflorescência, em pré-aplicação e aos 2, 4 e 6 dias após a aplicação dos inseticidas. Os produtos naturais Rotenat e NimI-Go apresentaram as melhores porcentagens médias de controle (respectivamente, $52,04 \%$ e $48,37 \%)$, seguidos pelo tiametoxam $(46,00 \%)$ e pelo Bio alho (23,07\%). Aos dois dias após a aplicação, o tiametoxam apresentou eficiência de $76,79 \%$, porém não manteve a eficiência ao longo das avaliações. As notas na escala de seletividade para o Bio Alho, Nim-I-Go, Rotenat e tiametoxam foram 2 (pouco tóxico), 3 (moderadamente tóxico), 3 e 4 (tóxico), respectivamente.
\end{abstract}

PALAVRAS-CHAVE: PRAGAS; INSETICIDAS; Vitis vinifera.

* $\quad$ Aluno do Programa de Pós-Graduação em Fitotecnia, Escola de Agronomia, Universidade Federal da Bahia (UFBA), Cruz das Almas, BA (e-mail: brasilmudas1@uol.com.br).

** Pesquisador, Doutora em Entomologia, Embrapa Semi-Árido, Petrolina, PE (e-mail: flavia@cpatsa.embrapa.br).

*** Professor, Doutor em Entomologia Escola de Agronomia, UFBA, Cruz das Almas, BA (e-mail: calfredo@ufba.br).

**** Aluno de Graduação em Agronomia, Universidade do Estado da Bahia (UNEB), Juazeiro, BA. 


\section{INTRODUÇÃO}

O pólo de irrigação Petrolina (PE) - Juazeiro (BA) constitui o maior centro produtor e exportador de uvas finas de mesa do Brasil. Contribui com $80 \%$ das exportações brasileiras de uva e desempenha importante papel social e econômico na região (AGRIANUAL, 2001; SILVA e CORREIA, 2000). Novos problemas fitossanitários têm sido observados com o aumento da área plantada, notadamente os relacionados com as pragas. No Vale do São Francisco, os tripes (Selenothrips rubrocinctus e Frankliniella sp.) estão entre as importantes pragas que danificam a videira.

$O$ adulto de $S$. rubrocinctus apresenta coloração geral preta ou marrom-escura. Os adultos e ninfas de $S$. rubrocinctus localizam-se na face inferior das folhas, principalmente nas proximidades das nervuras. Em função do ataque aparecem manchas amarelas cloróticas, que evoluem para o marrom. Quando o ataque é intenso ocorre a queima das folhas, podendo provocar desfolhamento parcial ou total da planta. $O$ adulto de Frankliniella sp. apresenta coloração variando do amarelo-claro ao marrom escuro. Nos frutos atacados (no local de postura) ocorre secamento e morte das células, formando lesão necrosada em forma de $Y$ e tornando os frutos imprestáveis para a comercialização (HAJI, ALENCAR e BARBOSA, 2002).

O tratamento químico é o método utilizado com maior frequência pelos produtores para o controle de tripes da videira. Contudo, o uso intensivo e indiscriminado desses produtos favorece 0 desenvolvimento de resistência aos inseticidas (HERRON e COOK, 2002), contamina o meio ambiente e reduz a população de inimigos naturais. Tal situação exige a adoção urgente de métodos de controle alternativos ao uso de agrotóxicos, que sejam menos poluentes, menos tóxicos ao homem e apresentem baixo poder residual.

Produtos naturais têm sido utilizados pelos produtores no controle de tripes em videira. São exemplos, o Nim I Go, óleo de nim (Azadirachta indica) emulsificado com extratos vegetais ativos de karanja (Pongamia glabra), a pimenta malagueta (Capsicum frutescens), a artemísia (Artemisia absythium), o annato (Bixa orellana); o Bio Alho, produto proveniente de extrato de alho (A. sativum) e o Rotenat, extrato de timbó (Lochocarpus floribundus) emulsionado (NIM..., [2004]). Para o controle químico, o tiametoxam, do grupo químico dos neonicotinóides, apresenta ação sistêmica, boa eficiência de controle para tripes e boa seletividade para inimigos naturais, que lhe conferem qualidades desejáveis no controle desses insetos (INSECTICIDE ... 2000; ANVISA, 2004).

O objetivo deste trabalho foi a avaliação de diferentes produtos naturais (Nim I Go, Bio Alho, Rotenat) e do tiametoxam no controle de tripes em videira e seus efeitos sobre inimigos naturais.

\section{MATERIAL E MÉTODOS}

O experimento foi conduzido em pomar comercial irrigado, no município de Juazeiro-BA (0924"S, 40-26"W, altitude $375 \mathrm{~m}$ ), utilizando-se plantas da cultivar Benitaka, com cinco anos de idade, irrigadas por microaspersão. Realizou-se avaliação prévia da população de tripes presente na área experimental. Aplicaram-se os produtos quando foram encontrados 2 ou mais tripes por inflorescência amostrada, que corresponde ao nível de ação para essa praga na cultura da videira, no Vale do São Francisco (HAJl et al., 2001).

Adotou-se o delineamento experimental de blocos ao acaso, em quatro repetições e cinco tratamentos: 1. Nim-I-Go (0,5\%); 2. Rotenat (0,5\%); 3. Bio Alho (0,3\%); 4. tiametoxam 250WG (20 g de produto comercial/100L) e 5. testemunha (sem inseticida). Adicionou-se o espalhante adesivo Agral na concentração de $0,03 \%$ em todos os produtos. A área experimental foi composta por 100 plantas, sendo 20 por tratamento, com 5 plantas por parcela. A área útil da parcela foi composta pelas três plantas centrais. Os tratamentos foram efetuadas no período entre 7 e 9 horas da manhã, com pulverizador Jacto-Arbus 1000 e volume de calda equivalente a $800 \mathrm{~L} / \mathrm{ha}$. Efetuaram-se quatro avaliações, sendo uma antes da aplicação dos produtos e aos 2, 4 e 6 dias após a aplicação. Foram amostrados, 
ao acaso, seis inflorescências por parcela útil, totalizando 24 inflorescências por avaliação/tratamento. Efetuou-se a amostragem pela batedura das inflorescências em bandeja plástica branca $(30 \mathrm{~cm} x$ $22 \mathrm{~cm} \times 7,5 \mathrm{~cm}$ ), conforme metodologia de HAJl et al. (2001), com posterior identificação e contagem dos tripes e inimigos naturais em microscópio estereoscópico.

As porcentagens de eficiência dos produtos foram calculadas pela fórmula de ABBOTT (1925). Já as porcentagens de redução populacional dos inimigos naturais foram mensuradas comparando-se a média do número de inimigos naturais na testemunha com a média do número de inimigos naturais nos tratamentos. As médias obtidas foram enquadradas na seguinte escala de seletividade: $1=$ inofensivo (<25\%), 2 = pouco tóxico $(25-50 \%), 3=$ moderadamente tóxico $(51-75 \%)$ e $4=$ tóxico $(>75 \%)$ (HASSAN et al., 1994). Para análise dos resultados aplicou-se o teste de Scott-Knot, utilizando o programa SisVar (FERREIRA, 2000).

\section{RESULTADOS E DISCUSSÃO}

Nas amostragens realizadas, 99\% dos tripes coletados foram identificados como Frankliniella sp. Aos dois e quatro dias após a aplicação dos produtos observou-se, nas parcelas tratadas, significativa redução no número de tripes/inflorescência. O tratamento com o tiametoxam 250WG apresentou maior controle aos dois dias, seguido em ordem decrescente pelo Rotenat, Nim-I-Go e Bio alho, os quais não apresentaram diferença significativa entre si. Aos quatro dias não foram constatadas diferenças significativas no número de tripes/inflorescência entre os produtos testados. Aos seis dias houve redução no número de tripes apenas quando se utilizou Rotenat e Nim-I-Go. As porcentagens de eficiência média dos produtos testados no controle de tripes aos 2, 4 e 6 dias foram, respectivamente: $47,7 \%, 46,7 \%$, e $55,8 \%$ para Nim-I-Go; $57,6 \%, 46,4 \%$ e $58,6 \%$ para Rotenat; $42,7 \%, 23,1 \%$ e $4,8 \%$ para o Bio Alho e 76,8\%, 34,3\% e 16,3\% para tiametoxam 250WG (Tabela 1). Resultado semelhante foi obtido para o óleo de Nim por SCHMUTTETER et al. (1995), citado por MARTINEZ (2002), no controle de F. schultzei, em amendoim, com redução da população e da transmissão de viroses. Contudo, BARBOSA et al. (2003) não observaram eficiência de óleo de Nim emulsionado (aplicado a $0,3 \%$ ) sobre tripes em inflorescência de mangueira. PEARSALL e HOGUE (2000) também relataram baixa eficiência de azadiractina (principal composto ativo do Nim) a $4 \%$ no controle de tripes em nectarina. De acordo com MARTINEZ (2002), a azadiractina atua de forma cumulativa e progressiva durante os sucessivos estádios de desenvolvimento dos insetos, podendo finalmente vir a causar a morte. Como as amostragens foram realizadas até os seis dias após a aplicação do produto, possivelmente, a eficiência do Nim poderá ser aumentada pelo seu modo de ação no inseto.

\section{TABELA 1 - EFEITO DOS TRATAMENTOS NO NÚMERO MÉDIO DE TRIPES/INFLORESCÊNCIA DE UVA E PORCENTAGEM DE CONTROLE (E) DOS INSETICIDAS TESTADOS EM AVALIAÇÕES REALIZADAS AOS 2, 4 E 6 DIAS APÓS A APLICAÇÃO (DAA) - JUAZEIRO-BA, 2003}

\begin{tabular}{|c|c|c|c|c|c|c|c|c|}
\hline \multirow[t]{3}{*}{ Tratamento } & \multicolumn{8}{|c|}{ Avalia ${ }^{a} 0$} \\
\hline & \multicolumn{2}{|c|}{2 DAA } & \multicolumn{2}{|l|}{$4 \mathrm{D}$} & \multicolumn{2}{|c|}{6 DAA } & Total & \multirow[t]{2}{*}{$E^{2}$} \\
\hline & $\begin{array}{c}\text { tripes }^{1} \\
\left(\mathrm{n}^{-}\right)\end{array}$ & $\begin{array}{l}E^{2} \\
(\%)\end{array}$ & $\begin{array}{c}\operatorname{tripes}^{1} \\
\left(\mathrm{n}^{\circ}\right)\end{array}$ & $\begin{array}{l}E^{2} \\
(\%)\end{array}$ & $\begin{array}{c}\text { tripes }^{1} \\
\left(\mathrm{n}^{\circ}\right)^{-}\end{array}$ & $\begin{array}{l}E^{2} \\
(\%)\end{array}$ & & \\
\hline$\overline{T e s}$ & $10,04 \mathrm{a}$ & - & $15,16 \mathrm{a}$ & - & $4,33 a$ & - & 709 & - \\
\hline $\mathrm{Nim}$ & 5,2 & 47,7 & $b$ & 46 & $1, \subseteq$ & 55,77 & 36 & 48,37 \\
\hline Rote & $4,25 \mathrm{~b}$ & 57,60 & $8,12 \mathrm{~b}$ & 46,4 & $1,79 \mathrm{~b}$ & 58,65 & 340 & 52,04 \\
\hline Bio Alho & $5,75 \mathrm{~b}$ & 42,73 & $11,67 b$ & 23,07 & $4,12 \mathrm{a}$ & 4,80 & 517 & 27,08 \\
\hline tiametoxam & $2,33 \mathrm{c}$ & 76,79 & $9,96 \mathrm{~b}$ & 34,34 & $3,62 \mathrm{a}$ & 16,35 & 382 & 46,00 \\
\hline
\end{tabular}

${ }^{1}$ Médias seguidas pela mesma letra na coluna não diferem estatisticamente pelo teste de agrupamento de Scott Knott. ${ }^{2}$ Porcentagem de controle (E) calculada pela fórmula de Abbott. 
Com a utilização de extrato de bulbo de Allium sativum, GUIRADO et al. (2000) obtiveram redução média de $87,8 \%$ no número de lesões da leprose do citros. O extrato atuou como deterrente alimentar para o ácaro Brevipalpus phoenicis, vetor dessa virose. Contudo, neste trabalho, não se observou boa eficiência no controle dos tripes da videira utilizando o produto comercial Bio Alho, proveniente de extrato de alho.

Constatou-se a presença de espécimes de crisopídeos e aracnídeos. O número médio de inimigos naturais na testemunha foi 0,21 , enquanto nas plantas tratadas com Nim-I-Go, Rotenat, Bio alho e tiametoxam 250WG foi, respectivamente, 0,$10 ; 0,11 ; 0,15$ e 0,05 . Tais resultados correspondem as notas 3, 3, 2 e 4 na escala de seletividade (Tabela 2). Segundo OLIVEIRA et al. (1988) produtos que apresentam efeito igual ou superior a $60 \%$ de mortalidade (notas 3 e 4 ) são considerados muito tóxicos aos inimigos naturais.

TABELA 2 - EFEITO DOS TRATAMENTOS NO NÚMERO MÉDIO DE INIMIGOS NATURAIS E SELETIVIDADE DOS INSETICIDAS, EM AVALIAÇÕES REALIZADAS AOS 2, 4 E 6 DIAS APÓS A APLICAÇÃO (DAA) - JUAZEIRO - BA, 2003

\begin{tabular}{lcccccccc}
\hline Tratamento & \multicolumn{1}{c}{2 DAA } & \multicolumn{4}{c}{ A DAalia ${ }^{2}$ o } & \multicolumn{2}{c}{ 6 DAA } & \multicolumn{2}{c}{ Mølia } \\
& IN & S & IN & S & IN & S & IN & S \\
& n. & (nota) & n. & (nota) & n. & (nota) & n. & (nota) \\
\hline Testemunha & 0,21 & - & 0,21 & - & 0,21 & - & 0,21 & - \\
Nim-I-Go & 0,13 & 2 & 0,08 & 3 & 0,08 & 3 & 0,10 & 3 \\
Rotenat & 0,04 & 4 & 0,21 & 1 & 0,08 & 3 & 0,11 & 3 \\
BioAlho & 0,13 & 2 & 0,17 & 1 & 0,17 & 1 & 0,15 & 2 \\
Tiametoxam & 0,00 & 4 & 0,08 & 3 & 0,08 & 3 & 0,05 & 4 \\
\hline
\end{tabular}

In = Inimigos naturais .

$\mathrm{S}=$ Seletividade

A baixa incidência de inimigos naturais no experimento (Tabela 2) pode ser explicada pela utilização de área comercial, na qual o controle das pragas é realizado rotineiramente. A baixa seletividade apresentada pelo produto Nim pode estar relacionada com a concentração utilizada $(0,5 \%)$. No trabalho relatado por BARBOSA et al. (2002), o produto Nim aplicado a 0,3\% apresentou nota média de seletividade igual a 1 . No que diz respeito ao tiametoxam, a compatibilidade da sua utilização em programas de manejo de pragas foram relatadas por outros autores, contrariando os resultados obtidos neste trabalho (OETTING e ANDERSON;1990; MULLINS e ENGLE, 1993; BARBOSA et al., 2003).

\section{CONCLUSÃO}

De maneira geral, os produtos testados revelaram baixa eficiência no controle dos tripes da videira. Os produtos naturais Nim-I-GO e Rotenat evidenciaram melhor porcentagem média de controle do que o Bio Alho. O tiametoxam apresentou boa eficiência de controle (76,79\%), aos dois dias após a aplicação, a qual não foi mantida ao longo das avaliações.

A baixa incidência de inimigos naturais no experimento pode ser explicada pela utilização de área comercial, na qual o controle das pragas é realizado rotineiramente. 


\begin{abstract}
EFFICIENCY OF NATURAL INSECTICIDES AND THIAMETHOXAM ON THE CONTROL OF THRIPS IN GRAPES AND SELECTIVITY TO NATURAL ENEMIES

The objective of this work was to verify the efficiency of natural insecticides and thiamethoxam on the control of Selenothrips rubrocinctus and Frankliniella sp. and their selectivity to natural enemies. The trial was conducted under field conditions, in an irrigated area of Juazeiro county, Bahia State, Brazil, in a five-years old vineyard with cv. Benitaka in a completly randomized block design with five treatments and four replicates. The treatments were: (1) Nim-I-Go $0.5 \%$; (2) Rotenat 0.5\%; (3) Bio Alho 0.3\%; (4) thiamethoxam 250WG (20 g c.p./100 L of water) and the control (untreated check). There were twelve plants per treatment, being evaluated number of thrips and natural enemies, at inflorescences before application and 2, 4 e 6 days after application. Rotenat and Nim-I-Go were the most effective, respectively, 52.04\% and $48.37 \%$ of control, followed by thiamethoxam $(46.00 \%)$ and Bio alho $(23.07 \%)$. Two days after application the efficiency of thiamethoxam was $76.79 \%$, however its average efficiency was low. The selectivity grades to Bio alho, Nim-I-Go, Rotenat and thiamethoxam were, respectively, 2 (slightly harmful, 3 (moderately harmful), 3 and 4 (harmful).
\end{abstract}

KEY-WORDS: PESTS; PESTICIDES; Vitis vinifera.

\title{
REFERÊNCIAS
}

1 ABBOTT, W. S. A method of computing the effectiveness of an insecticide. Journal of Economic Entomology, Lanham, v. 18, p. 265-267, 1925.

2 AGRIANUAL. Anuário estatístico da agricultura brasileira. São Paulo: FNP, 2001. 487 p.

3 ANVISA. Sistema de informações sobre agrotóxicos. Disponível em: <http://www4.anvisa.gov.br/agrosia/asp/ default.asp.> Acesso em: 03 set. 2004.

4 BARBOSA, F. R.; FERREIRA, R. G.; KIILL, L. H. P.; SOUZA, E. A. de; MOREIRA, W. A ALENCAR, J. A. de; HAJI, F. N. P. Nível de dano, plantas invasoras hospedeiras, inimigos naturais e controle do psilídeo da goiabeira (Triozoida sp.) no Submédio São Francisco. Revista Brasileira de Fruticultura, Jaboticabal, v. 25, n. 3, p. 425-428. 2003.

5 BARBOSA, F. R.; SOUZA, E. A. de; SILVA, C. S. B. da; MOREIRA, W. A.; ALENCAR, J. A. de; HAJI, F. N. P. Eficiência de inseticidas no controle de tripes em mangueira e efeito sobre inimigos naturais. In: CONGRESSO BRASILEIRO DE FRUTICULTURA, 17., 2002, Belém, PA: Anais... Belém:SBF, 2002. CD-ROM.

6 FERREIRA, D. F. Análises estatísticas por meio do SISVAR (Sistema para Análise de Variância), para Windows 4.0. In: REUNIÃO ANUAL DA REGIÃO BRASILEIRA DA SOCIEDADE INTERNACIONAL DE BIOMETRIA, 45., 2000, São Carlos. Anais... São Carlos: UFSCarlos, 2000. p. 255-258.

7 GUIRADO, N; NOGUEIRA, N. de L.; SILVA, A. C. da; ROSSI, M. L. Extratos vegetais no controle da leprose dos citros. In: CONGRESSO BRASILEIRO DE DEFENSIVOS AGRÍCOLAS NATURAIS, 1., 2000, Fortaleza. Resumos... Fortaleza: ACECI, 2000.

8 HAJI, F. N. P.; ALENCAR, J. A. de; BARBOSA, F. R. Pragas. In: LIMA, M. F.; MOREIRA, W. A. (Ed.). Uva de mesa: fitossanidade. Brasília: Embrapa Comunicação para Transferência de Tecnologia, 2002. p. 53-63 (Frutas do Brasil, 14).

9 HAJI, F. N. P.; MOREIRA, A. N.; FERREIRA, R. C. F.; LOPES, L. M. da M.; ALENCAR, J. A. de; BARBOSA, F. R. Monitoramento e determinação do nível de ação para tripes na cultura da uva. Petrolina: Embrapa SemiÁrido, 2001. 8 p. (Embrapa Semi-Árido. Circular Técnica, 70).

10 HASSAN, S. A.; BIGLER, F.; BOGENSCHUTZ, H.; BOLLER, E.; BRUN, J.; CALIS, J. N. M.; COREMANS-PELSENEER, J.; DUSO, C.; GROVE, A.; HEIMBACH, U.; HELYER, N.; HOKKANEN, H.; LEWIS, G. B.; MANSOUR, F.; MORETH, L.; POLGAR, L.; SAMSOE-PETERSEN, L.; SAUPHANOR, B.; STAUBLI, A.; STERK, G.; VAINIO, A.; VEIRE, M. van de; VIGGIANI, G.; VOGT, H. Results of the six pesticide testing programme of the IOBC/WPRS-Working group pesticides and beneficial organisms. Entomophaga, Paris, v. 39, p. 107-119, 1994.

11 HERRON, G. A.; COOK, D. F. Initial verification of the resistance management strategy for Frankliniella occidentalis (Pergande) (Thysanoptera: Thripidae) in Australia. Australian Journal of Entomology, Canberra, v. 41, p. 182, 2002.

12 INSECTICIDE technology basket. Basel: Novartis, 2000. (Acompanha 1 CD-Rom).

13 MARTINEZ, S. S. O nim: Azadirachta indica: natureza, usos múltiplos, produção. Londrina: IAPAR, 2002. 142 p.

14 MULLINS, J. W.; ENGLE, C. E. Imidacloprid (BAY NTN 33893): novel chemistry for sweetpotato whitefly control in cotton. In: BELTWIDE COTTON PRODUCTION CONFERENCES, 2., 1993, Tennessee. Proceedings... Tennessee: National Cotton Council of America, 1993. p. 719-720. 
15 NIM I GO: controle natural de insetos - nova fórmula com extratos naturais. óleo de nim emulsificado (Azadirachta indica A. Juss.) com extratos vegetais ativos. [S.I.]: Agro Ecologic, [2004]. 1 folder.

16 OETTING, R. D.; ANDERSON, A. L. Imidacloprid for control of whiteflies, Trialeurodes vaporariorum and Bemisia tabaci, on greenhouse grown poinsettias. In: BRIGTHON CROP PROTECTION CONFERENCE - PEST AND DISEASE, 1., 1990, Thornton Heath. Proceedings... Thornton Heath, 1990. p. 367-372.

17 OLIVEIRA, E. B. de; GAZZONI, D. L.; CORSO, I. C.; VILLAS BÔAS, G. L.; HOFFMANN-CAMPO, C. B. Pesquisa com inseticidas em soja: sumário dos resultados alcançados entre 1975 e 1987. Londrina, PR: Embrapa-CNPSo, 1988. 260 p. (Embrapa-CNPSo, Série Documentos, 30).

18 PEARSALL, I. A.; HOGUE, E. J. Use of azadirachtin as a larvicide or feeding deterrent for control of western flower thrips in orchard systems. Phytoparasitica, Bet Dagani, v. 28, 2000. Disponível em: <http:// www.phytoparasitica.org.> Acesso em: 24 maio de 2004.

19 SILVA, P. C. G.; CORREIA, R. C. Caracterização social e econômica da videira. In: LEÃO, P.C.S.; SOARES, J.M. A viticultura no semi-árido brasileiro. Petrolina: Embrapa Semi-Árido, 2000. p. 19-32. 